\title{
Editorial
}

\section{Football et violence : pour une approche pluridisciplinaire}

\author{
W. Nuytens et N. Penin \\ Laboratoire Sherpas, Université d'Artois, Faculté des sciences du sport, chemin du marquage, 62800 Liévin, France
}

Les violences dans et autour des sports font partie de ces phénomènes dont la compréhension nécessite une approche pluridisciplinaire. Cela s'explique d'abord par leur caractère pluriel : elles peuvent être physiques, verbales, symboliques portant ainsi atteinte à l'intégrité d'autrui dans son entièreté. On doit ensuite relever combien il est ardu de définir ce qu'est la violence selon que l'on se place du côté des victimes ou des contrevenants, des institutions sportives et de leur réglementation voire de systèmes normatifs moins spécialisés. C'est mécaniquement, enfin, qu'on ne compte pas les violences avec précision surtout si l'on songe aux processus de stigmatisation s'imposant en conséquence. La somme de tels attributs explique peut-être le recours obligé aux cadrages théoriques d'envergure soit, pour le dire autrement, l'audience relative de travaux proprement empiriques. Cette configuration est dommageable parce que c'est en valorisant ces derniers qu'émergent des modes d'intervention efficaces, réalisables sur des terrains qui manquent de ressources à cet égard.

C'est que sans être particulièrement répandues les violences perturbent l'ordre sportif, défont des vocations, fragilisent institutions et pratiques. Elles marquent également, parfois en profondeur, les victimes et plus rarement de prétendus auteurs. C'est dire combien les violences constituent une menace pour le sport surtout si celles et ceux qui l'encadrent, le pratiquent et le dirigent délaissent des outils précis d'intervention au profit d'une séduisante mais inopérante doxa. C'est donc à une démonstration des intérêts de travaux proches du « sol raboteux » qu'est consacré ce dossier : saisir la complexité du couple sport et violence à partir du cas exemplaire du football amateur et au plus près de ce qui se joue, est la posture défendue ici. Voilà une stratégie que renforce la persistance des violences dans les pratiques sportives; un encombrant démenti à la puissante théorie du « procès de civilisation ». Mais comment tenir une telle position sans craindre les pertes d'envergure qu'induisent ces gains de précisions?

C'est en convoquant diverses approches que ce numéro entend traiter cette gageure : faire varier les échelles de questionnement, les terrains étudiés, les sensibilités théoriques, les ancrages scientifiques de sorte que l'ensemble des connaissances présentées puissent faire système. Se complètent ainsi contributions de sociologues, géographe ou psychologues qui posent le regard sur des territoires, des clubs, des situations, des interactions, des processus cognitifs. Se dessine alors une posture épistémologique traduite en actes, d'organisation centripète, accrochée au plus près et soucieuse de participer au changement social.

La contribution de José Chaboche est celle d'un géographe s'intéressant aux «territoires de la violence ». À l'échelle d'un département il met en évidence non seulement les espaces les plus concernés, mais aussi des « configurations territoriales » propices ou non à la contention ou à l'apparition des violences. L'auteur démontre ainsi que la violence est aussi une affaire de lieu(x) contenant un jeu complexe de médiations et de logiques de cristallisations. Dans le second texte Jean-Louis Bergez et Paul Carry placent la question de la reconnaissance sociale au cour de l'explication des violences sportives. Leur enquête sociologique montre comment, dans des conditions de pratique précaires, des formes de reconnaissances qui ne sont pas seulement « sportives » peuvent contribuer à la pacification des comportements. Comment l'absence de reconnaissance aussi peut constituer un puissant catalyseur de tensions dans les situations. Ces auteurs parviennent progressivement à souligner la responsabilité des acteurs locaux et des politiques de clubs. C'est justement sur les situations de match que se concentre la livraison d'Alain Traclet, Orlan Moret, Philippe Romand, Fabien Ohl et Alain Clémence. Elle éclaire l'influence de variables chronologiques et topographiques sur l'apparition de conduites violentes. Elle permet ainsi de repérer quelques endroits, moments ou évolutions du score particulièrement « sensibles ». Construite à partir d'une démarche comparative et depuis une procédure élaborée de codage des faits, cette recherche parvient à théoriser l'épineuse ambiguité entre les violences instrumentales et les violences hostiles. Le quatrième texte rend compte d'une enquête exploratoire de Géraldine Rix-Liévre et Vincent Genebrier. Elle concerne les interactions 
entre joueurs et arbitres. Celle-ci indique d'abord qu'il existe de fréquentes disjonctions entre la perception des situations par les arbitres et par les joueurs. Ce constat laisse ainsi penser que les interactions diverses et répétées entre arbitres et joueurs pourraient participer à la réduction de l'écart des «points de vue»: en construisant des modalités partagées d'interactions on contribuerait à pacifier les matchs. Au cœur de l'interaction et de processus de régulation, la contribution emmène le lecteur dans les mécanismes qui font et défont la dissymétrie des relations c'est-à-dire au plus près de ce qui crée l'émergence (ou l'absence) de heurts. Il est aussi question de disjonction dans le dernier article de ce numéro. Lionel Faccenda, Nathalie Pantaléon et Thierry Long rendent compte d'une enquête visant à éprouver l'influence du sentiment d'injustice sur le recours aux violences. Les psychologues montrent primo que l'agression est bien plus tolérée par les joueurs si elle fait suite à ce qui est perçu comme une injustice arbitrale, et secundo que la sensibilité à l'injustice - variable intraindivuelle - présente un puissant pouvoir prédicteur. Au total, l'ensemble de ces travaux prouve le caractère heuristique d'une approche écologique associant les échelles d'observations, multipliant les énoncés de discriminants et de variables. Le fait qu'il soit possible d'en apprécier la portée en inversant l'ordre des lectures, soit en partant d'une économie émotionnelle pour finir par l'économie territoriale, constitue de ce point de vue une preuve notable. Nous espérons effectivement que les lecteurs trouveront ici des éléments d'explications qui dépassent le seul périmètre de la première pratique sportive des Français. 